\title{
Acute Retropharyngeal Abscess Masquerading as Meningitis
}

\author{
Pirabu Sakthivel, ${ }^{1}$ Rijendra Yogal, ${ }^{1}$ Hitesh Verma, ${ }^{1}$ Anil Saini, ${ }^{1}$ Ashwin Chandran ${ }^{1}$ \\ 'Department of ENT, All India Institute of Medical Sciences, New Delhi, India.
}

\section{ABSTRACT}

Retropharyngeal abscess is a potentially serious deep neck space infection occurring more frequently in children than in adults. The clinical picture of RPA is highly variable with paucity of physical findings. Prompt diagnosis of RPA especially in infants is mandatory to prevent potential fatal complications including airway obstruction. The diagnosis of RPA should be based on high index of clinical suspicion with supportive imaging studies like lateral X-ray of neck and CT.

We present a case of acute retropharyngeal abscess which was initially misdiagnosed as meningitis and led to airway obstruction. This case is reported to create awareness among emergency physicians, paediatricians and otolaryngologists to have high index of suspicion in diagnosing RPA especially in infants.

Keywords: retropharyngeal abscess; meningitis; deep neck infection.

\section{INTRODUCTION}

Retropharyngeal abscess (RPA) is a potentially life threatening deep neck space infection occurring more frequently in children than in adults. Complications of RPA include airway obstruction, mediastinitis, jugular vein thrombosis, carotid artery stenosis or rupture, atlanto-axial subluxation, cervical osteomyelitis, spinal cord abscess and meningitis. Mortality rates of $10 \%$ and complication rates of $43 \%$ have been reported. ${ }^{1,2}$ Early diagnosis is necessary to prevent the development of complications but because of their relative infrequency, variability in presenting symptoms and paucity of physical findings, they almost always pose a diagnostic challenge to emergency physicians, pediatricians and otolaryngologists. Hence, diagnosis of RPA should be based on high index of clinical suspicion with supportive imaging studies like lateral X-ray of neck and CT. Prompt surgical drainage, either transoral or transcervical has been the classically accepted treatment for this condition, but given improvements in antibiotic therapy and paediatric critical care in recent times, the optimal management today is still a matter of debate..$^{3,4}$

We present a case of acute retropharyngeal abscess which was initially misdiagnosed as meningitis. This case is reported to create awareness among emergency physicians, pediatricians and otolaryngologists to have high index of suspicion in diagnosing RPA especially in infants.

\section{CASE REPORT}

A 11-month-old male baby presented to Paediatrics department with complaints of fever, irritability and poor intake of feeds for 3 days duration following an episode of upper respiratory tract infection. The child was febrile $\left(101.4^{\circ} \mathrm{F}\right)$ with neck stiffness and a clinical diagnosis of meningitis was made. The child

Correspondence: Dr. Rijendra Yogal, Department of ENT, All India Institute of Medical Sciences, New Delhi, India. Email: rijendrayogal@gmail.com, Phone: +919654901965. 
was admitted, and therapy for possible bacterial sepsis was initiated with ceftriaxone and metronidazole. Meanwhile, cultures of blood, urine and CSF were found to be negative. After 3 days, there was no clinical improvement and child had worsening of symptoms. A lateral X-ray neck film (Figure 1) showed thickened soft tissues in the nasopharynx with marked anterior pharyngeal deviation and obliteration of nasal airway without any air pockets. The child was immediately referred to our department for further management. On examination, the child had severe stertor and marked bulge was seen on the posterior pharyngeal wall. There was difficulty in neck extension and there was no neck swelling. Clinical diagnosis of acute retropharyngeal abscess/cellulitis was made. As the child had severe stertor, CT under sedation was not done and emergency drainage was planned. The oropharyngeal airway was obstructed, hence an emergency tracheotomy was done and around $25 \mathrm{ml}$ of frank thick pus was aspirated (Figure 2). Intraoral incision and drainage was done and entire abscess was drained. The child was afebrile from the second postoperative day and pus culture was sterile. CECT neck on post-operative day 7 was normal and the child was discharged in stable condition after decannulation.

\section{DISCUSSION}

RPA is a serious and occasionally life-threatening deep neck infection, because of the anatomic location and the potential to obstruct the upper airway. RPAs are thought to occur as a consequence of infections of the nasopharynx, paranasal sinuses, or middle ear and these infectious foci extend to lymph nodes located in the space between the posterior pharyngeal wall and the prevertebral fascia. These lymph nodes likely atrophy after the first 3 or 4 years of life although some may persist till puberty. ${ }^{5}$ More than $75 \%$ of cases occur in children younger than 5 years. Male predominance in retropharyngeal abscess is a well-recognized phenomenon but the exact cause is not known. ${ }^{6}$ Fever, poor intake of feeds and neck pain are common presenting symptoms while neck swelling, drooling and torticollis are common signs. RPA presents similar to epiglottitis with fever, stridor, drooling, and sometimes meningismus, and it was recently dubbed the "epiglottitis of the new millennium". ${ }^{7}$ The small size of the pediatric oropharynx, with pooling of secretions in the pharynx consequent to swelling, makes assessment of the posterior pharyngeal wall extremely difficult. Hence, a retropharyngeal bulge may not be evident in infants and young children. Airway symptoms are unusual but as the pus collection increases, it is more likely to result in airway symptoms. Because respiratory difficulty and stridor are late findings, limited neck mobility should be the early potential diagnostic clinical clue for diagnosing
RPA. The limitation in meningitis usually involves neck flexion whereas in RPA the limitation will be for neck extension. ${ }^{8}$ Heightened suspicion for RPA should be kept when the child does not fully extend his or her neck to look up ('Bolte's sign'). According to previous literature, seven patients underwent lumbar puncture for evaluation of meningitis presumably because of the presence of fever and a stiff neck as in our case..$^{1,6}$

If an RPA is suspected a lateral airway X-ray may be useful as a screening study. But it is difficult to acquire an adequate extension film on a child with painful torticollis and often the films are obtained with the child in supine position and with their relatively larger head this causes the film to be obtained in flexion. In addition, parapharyngeal infections are not visible on a lateral X-ray film. Unless gas is present, it does not discriminate between retropharyngeal space cellulitis and abscess formation. ${ }^{3}$

Widespread availability of CT scanning facility and increased familiarity with the clinical presentation may obviate the need for a 'screening' lateral neck radiograph. The sensitivity of CT for diagnosing RPA has been reported to be $88-91 \% .{ }^{1}$ A study by Kirse and Roberson suggests that scalloping of the abscess wall may more accurately predict pus than other findings like low density numbers, the presence of air or fluid, and rim enhancement. ${ }^{9}$ Ultrasound is not routinely performed for diagnosing RPA. There is one study suggesting that an increased distance from the internal carotid artery to the cervical vertebrae could be used as a reliable criterion, however, the study included only five cases. ${ }^{9}$

Treatment of children who have a diagnosis of RPA is still evolving. Traditional management of RPA has been surgical drainage of the pus collection, with intraoral incision and drainage currently being the preferred technique. External cervical drainage is reserved for abscesses that have extended to contiguous deep neck spaces such as the parapharyngeal space, multiloculated abscesses and persistence or recurrence of abscess following intraoral drainage. ${ }^{9}$ With the paucity of reported failures after antibiotic treatment alone, a trial of antibiotics should be considered in all cases of retropharyngeal cellulitis and some cases of RPA in which the abscess is small and when there is no evidence of airway compromise. ${ }^{6}$

The management of the child with associated airway symptoms is always a challenge. A high rate of emergency tracheotomy $(12-37 \%)$ is reported in those patients with airway symptoms, with some authors performing a tracheotomy under local anaesthetic before drainage of the abscess. ${ }^{3,11}$ In cases where there is no airway compromise, Dawes et al. advised 
for short-term postoperative intubation and ventilation for all children with retropharyngeal abscess. ${ }^{1}$

RPA should be considered in all young patients with limited range of motion of the neck and fever, even in the absence of any respiratory findings. Delay in clinical diagnosis and management may lead to potentially life threatening complications.

\section{Conflict of Interest: None.}

Consent: JNMA Case Report Consent Form was signed by the patient and the original is attached with the patient chart.

\section{REFERENCES}

1. Dawes LC, Bova R, Carter P. Retropharyngeal abscess in children. ANZ J Surg. 2002 Jun;72:417-20. [PubMed]

2. Gianoli GJ, Espinola TE, Guarisco JL, Miller RH. Retropharyngeal space infection: Changing trends. Otolaryngol Head Neck Surg. 1991 Jul;105(1):92-100. [PubMed]

3. Daya H, Lo S, Papsin BC, Zachariasova A, Murray $\mathrm{H}$, Pirie J, et al. Retropharyngeal and parapharyngeal infections in children: the Toronto experience. Int J Pediatr Otorhinolaryngol. 2005 Jan;69(1):81-6. [PubMed]

4. Page NC, Bauer EM, Lieu JE. Clinical features and treatment of retropharyngeal abscess in children. Otolaryngol Head Neck Surg. 2008 Mar;138(3):300-6. [PubMed]

5. Asmar BI. Bacteriology of retropharyngeal abscess in children. Pediatr Infect Dis J. 1990;9(8):595-7. [PubMed]
6. Craig FW, Schunk JE. Retropharyngeal abscess in children: clinical presentation, utility of imaging, and current management. Pediatrics. 2003 Jun;111:1394-8. [PubMed]

7. Lee SS, Schwartz RH, Bahadori RS. Retropharyngeal abscess: epiglottitis of the new millennium. J Pediatr. 2001 Mar;138(3):435-7. [PubMed]

8. Morrison JE, Pashley NRT. Retropharyngeal abscesses in children: a 10-year review. Pediatr Emerg Care. 1988 Mar;4:9-11. [PubMed]

9. Kirse DJ, Roberson DW. Surgical management of retropharyngeal space infections in children. Laryngoscope. 2001 Aug;111(8):1413-22. [PubMed]

10. Chao HC, Chiu CH, Lin SJ, Lin TY. Colour Doppler ultrasonography of retropharyngeal abscess. J Otolaryngol. 1999 Jun;28(3):138-41. [PubMed]

11. Gidley PW, Ghorayeb BY, Stiernberg CM. Contemporary management of deep neck space infections. Otolaryngol Head Neck Surg. 1997 Jan;116(1):16-22. [PubMed] 